\title{
New first integral for twisting type-N vacuum gravitational fields with two non-commuting Killing vectors
}

\author{
F J Chinea \\ Departamento de Física Teórica II, Facultad de Ciencias Físicas, Universidad \\ Complutense, 28040 Madrid, Spain
}

\begin{abstract}
A new first integral for the equations corresponding to twisting type- $\mathrm{N}$ vacuum gravitational fields with two non-commuting Killing vectors is introduced. A new reduction of the problem to a complex second-order ordinary differential equation is given. Alternatively, the mentioned first integral can be used in order to provide a first integral of the second-order complex equation introduced in a previous treatment of the problem.
\end{abstract}

\section{Introduction}

It is known from the peeling-off property that the dominant term in an asymptotic expansion of the gravitational field is of algebraic type N. Thus, such fields can be considered as containing the main features of gravitational radiation far from the sources. However, twist-free type- $\mathrm{N}$ fields are known to contain unavoidable singularities. The only explicitly known type-N vacuum field with twist [2] suffers from similar problems. In the quest for considering a purely type- $\mathrm{N}$ field throughout the whole spacetime as a theoretical model for realistic ones produced by compact sources, there remains the hope that twisting fields with the appropriate behaviour may eventually be found. In spite of numerous efforts, no new explicit fields are known. The information gathered on twisting type- $\mathrm{N}$ fields, especially when additional symmetries are considered, can be seen in references such as [1]-19.

The maximum number of independent isometries for such gravitational fields can be shown to be two, in which case the corresponding Lie algebra is necessarily non-Abelian [6]. Although the approach used in [2] was based on algebraic properties of some exterior form equations, the solution found by Hauser turns out to possess one Killing vector and one homothetic Killing vector. This naturally led to a more detailed investigation of symmetries of such a type. The special case of two proper Killing vectors was treated in 
[10], where a reduction of the problem to a second-order complex differential equation was shown. By further manipulation, it was also shown there that the problem can be reduced to the study of a single third-order real equation. In what follows, we first discuss in the next section the main features and results of the previous treatment. Within this framework, we then introduce in the final section a new first integral of the field equations; it gives rise to a more direct reduction in terms of the original variables appearing in the metric, while it can also be used as a (previously unnoticed) first integral of the second-order complex equation considered in [10]. Our aim in pursuing the present approach is twofold: firstly, to cast the problem in a form more amenable to numerical treatment, and secondly, to open the way for the intriguing possibility of final reduction to a second-order real equation.

\section{Twisting type-N vacuum fields with two non-commuting Killing vectors}

The reduction of the field and symmetry equations described in this section was carried out in [10] by means of a compact version of the Cartan structure equations [20], in terms of an $s l(2, C)$-valued 1 -form connection; the empty space Einstein equations then take a particularly simple form. The conditions for the existence of two non-commuting Killing vectors and the final expression for such Killing fields were performed at the tetrad rather than at the metric level, by using techniques modelled on similar ones in gauge theories [9]. Based on [6], it was required that the Lie algebra of isometries took the form

$$
\left[\xi_{1}, \xi_{2}\right]=\xi_{1}
$$

The connection 1-form was chosen to be in a gauge of maximum simplicity, following the gauge used in [2]. Such a gauge is compatible with the requirement that the field be of type N. It leads to the vanishing of all components of the Weyl spinor, except for $\Psi_{4}$. The metric is expressed in terms of the null tetrad $\{l, n, m, \bar{m}\}$ (where $l$ and $n$ are real 1 -forms, while $m$ is a complex one, with complex conjugation being denoted by a bar) by means of the usual relation

$$
g=-2 l \otimes_{s} n+2 m \otimes_{s} \bar{m}
$$

where $\otimes_{s}$ means the symmetrized tensor product. The 1 -form $l$ is the repeated principal null eigenform, and its twist is assumed not to vanish. The four independent spacetime coordinates are $\{u, \sigma, \zeta, \bar{\zeta}\}$, where $u$ and $\sigma$ are real, while $\zeta$ is complex. Details of the procedure need not concern us here; they are given in [9] and [10]. The Killing vectors can be shown to be

$$
\xi_{1}=i \partial_{\zeta}-i \partial_{\bar{\zeta}}
$$


and

$$
\xi_{2}=\zeta \partial_{\zeta}+\bar{\zeta} \partial_{\bar{\zeta}}
$$

The tetrad can be expressed as $\dagger$

$$
\begin{gathered}
l=(\zeta+\bar{\zeta}) \mathrm{d} u+D \mathrm{~d} \zeta+\bar{D} \mathrm{~d} \bar{\zeta} \\
n=(\zeta+\bar{\zeta})^{-1} \mathrm{~d} \sigma+(-\sigma+F \bar{D}-M)(\zeta+\bar{\zeta})^{-2} \mathrm{~d} \zeta+(-\sigma+\bar{F} D-\bar{M})(\zeta+\bar{\zeta})^{-2} \mathrm{~d} \bar{\zeta} \\
m=\bar{D}^{-1} \bar{M} \mathrm{~d} u-\sigma(\zeta+\bar{\zeta})^{-1} \mathrm{~d} \zeta+\bar{M}(\zeta+\bar{\zeta})^{-1} \mathrm{~d} \bar{\zeta},
\end{gathered}
$$

where $D, M$ and $F$ are complex functions of the real independent variable $u$ only. Due to the first Bianchi identities, the requirement that the resulting metric be of type N, the conditions for the existence of the Killing fields, and the vacuum field equations, these functions are subject to the following system of equations:

$$
\begin{aligned}
& M_{u}=-F \\
& F_{u}=-2 F \bar{D}^{-1}, \\
& D_{u}=1-D^{-1} M, \\
& 2 M-2 \bar{M}-2 F \bar{D}+2 \bar{F} D+F \bar{M}-\bar{F} M=0,
\end{aligned}
$$

where the subscript denotes a derivative with respect to $u$. Note that (11) takes the form of a first integral of the previous equations (8)-(10) (in the sense that the derivative of its left-hand side with respect to $u$ vanishes when those first three equations are taken into account). Two points should be stressed, however; firstly, (11) appears by itself as one of the combined field and symmetry equations, rather than being searched for; and secondly, its right-hand side is not an arbitrary constant, but has to take the specific value zero.

If we define the new independent variable $s=\ln v$, with

$$
v(u)=\int F(u) \bar{F}(u) \mathrm{d} u,
$$

and the new complex dependent variable $z$ as

$$
z=2 v^{-1} M
$$

$\dagger$ The tetrad was denoted by $k, m, t, \bar{t}$ in [10]; the correspondence with the notation used here is the following: $k \rightarrow-l, m \rightarrow n, t \rightarrow m$. 
then the set of equations (8)-(11) reduce to the single second-order autonomous ordinary differential equation

$$
\ddot{z}+\dot{z}+\frac{2(z+\dot{z})^{2}(\bar{z}+\dot{\bar{z}})}{z \bar{z}+\bar{z} \dot{z}+\dot{z}}=0,
$$

where dots denote derivatives with respect to the real independent variable $s$. By rewriting the set (8)-(11) as equations in the independent variable $v$, it can be easily seen that $M, D$ and $F$ are explicitly given algebraically in terms of $z, \bar{z}$ and their first derivatives (the second and successive derivatives of $z$ and $\bar{z}$ can be expressed in terms of the first-order ones by means of (14) and its complex conjugate). Finally, the original independent variable $u$ is simply given by a quadrature, corresponding to the inversion of (12). The details are given in [10].

By further manipulation of (14) (involving a Legendre transformation and the compatibility conditions for an associated linear system), it was shown in [10] that the problem can be reduced to a single, third-order, real ordinary differential equation. Similar techniques have been subsequently used in [12] and [13] in the more general case of the existence of one Killing vector and one homothetic Killing vector, showing also a reduction to a final, third-order equation. It is rather remarkable that the simplest such equation was found in 12 for one specific case of the existence of a proper homothetic Killing vector (i.e. a case not reducible to the situation considered in the present paper). The reduction to third-order equations and the relations among different approaches have been further investigated in [15], [17], and [18].

For completeness, the remainder of this section will be used for recording some important quantities that appear in the present problem. They can be computed in a straightforward fashion from the tetrad (5)-(7) (taking into account (8)-(11) when derivatives are involved), especially if one uses some computer algebra software. The only non-vanishing spin coefficients are the following:

$$
\begin{aligned}
& \lambda=\frac{F \bar{D}}{(\sigma \bar{D}+\bar{M} D)(\zeta+\bar{\zeta})} \\
& \nu=\frac{F \bar{M}}{(\sigma \bar{D}+\bar{M} D)(\zeta+\bar{\zeta})^{2}} \\
& \rho=-\frac{\bar{D}(\zeta+\bar{\zeta})}{(\sigma \bar{D}+\bar{M} D)} \\
& \tau=-\frac{\bar{M}}{(\sigma \bar{D}+\bar{M} D)} .
\end{aligned}
$$


As $\rho=\theta+i \omega$, where $\theta$ is the expansion and $\omega$ is the twist of the congruence defined by $l$, the twist is given by the following expression:

$$
\omega=\frac{i}{2}(\zeta+\bar{\zeta}) \frac{M \bar{D}^{2}-\bar{M} D^{2}}{(\sigma \bar{D}+\bar{M} D)(\sigma D+M \bar{D})} .
$$

All components of the Weyl spinor vanish, except for $\Psi_{4}$, as corresponds to the type-N character in the gauge we are using. The latter is given by

$$
\Psi_{4}=\frac{2 F}{(\sigma \bar{D}+\bar{M} D)(\zeta+\bar{\zeta})^{2}} .
$$

From (19) and (20), we should keep in mind for non-triviality that

$$
\text { non-flat spacetime } \Longleftrightarrow F \neq 0,
$$

while

$$
\text { non-vanishing twist } \Longleftrightarrow M \bar{D}^{2}-\bar{M} D^{2} \neq 0 .
$$

Note that the condition (21) is precisely what is required for the coordinate change (12) to be defined.

As the twist measures the failure of the 1-form $l$ to be Frobenius integrable, it is sometimes convenient to consider the following alternative expression to (19):

$$
\mathrm{d} l \wedge l=-\frac{1}{D \bar{D}}\left(M \bar{D}^{2}-\bar{M} D^{2}\right) \mathrm{d} u \wedge \mathrm{d} \zeta \wedge \mathrm{d} \bar{\zeta}
$$

\section{New first integral and reduction}

The present author realized recently that the system of equations (8)-(10) admit the following first integral, which went unnoticed in [10]:

$$
F \bar{D}^{2}+\bar{F} D^{2}-2 M \bar{M}=k,
$$

where $k$ is an arbitrary real constant. As an alternative to the reduction to a final secondorder complex equation given in [10] (equation (14) above), in the manner discussed in the previous section, the following new possibility presents itself: from (10) one can write

$$
M=D-D D_{u},
$$

and then, upon differentiation of (25) with respect to $u$ and substitution into (8), $F$ can be expressed as

$$
F=D D_{u u}+D_{u}^{2}-D_{u} .
$$

By substituting $M, \bar{M}, F$ and $\bar{F}$ as given by (25) and (26) (and their complex conjugates) into the constraint equation (11) and the first integral (24), one ends up with two real 
equations where only $D, \bar{D}$, and their derivatives up to the second order appear. They can be solved for $D_{u u}$ (and its complex conjugate). The result is the following:

$$
D_{u u}=\frac{A}{B},
$$

where

$$
\begin{aligned}
& A=-\bar{D}^{2} D_{u}^{3}+D \bar{D} \bar{D}_{u} D_{u}^{2}-3 D \bar{D} D_{u}^{2}+D \bar{D} D_{u} \bar{D}_{u} \\
& -2 D^{2} D_{u}+\bar{D}^{2} D_{u}+D \bar{D} D_{u}+k D_{u}+2 D^{2}+k
\end{aligned}
$$

and

$$
B=D \bar{D}\left(D \bar{D}_{u}+\bar{D} D_{u}+D+\bar{D}\right) .
$$

Finally, there remains equation (9), which is third order in $D$. But third-order derivatives can be obtained from (27). When this is done, (9) is satisfied identically. In other words, the only equation to be solved is precisely the second-order complex equation (27). While this equation is rather complicated, it has the following property: it determines $D$ as a function of the original independent variable $u$, without the need of transformations such as (12). It seems that this will allow for a more direct numerical treatment of the twisting type-N problem, as compared with an approach based on (14) or its associated third-order real equation. On the other hand, the new integral (24) can also be used in connection with (14): By carrying out the changes discussed in the previous section, one ends up with additional information about (14). Namely, it admits the following first integral:

$$
I_{0}=\frac{\mathrm{e}^{2 s}}{\bar{z}+\dot{\bar{z}}}(z \bar{z}+z \dot{\bar{z}}+\dot{\bar{z}})^{2}+\frac{\mathrm{e}^{2 s}}{z+\dot{z}}(z \bar{z}+\bar{z} \dot{z}+\dot{z})^{2}+4 \mathrm{e}^{2 s} z \bar{z},
$$

where $I_{0}$ is an arbitrary real constant. Numerical treatment of the problem with the aid of the new first integral is currently under consideration [21]. Finally, the following remark can be made: As the second-order complex differential equation (14) was shown to be reducible to a third-order real one, there is some hope that the new first integral may result in the reduction to a second-order real differential equation.

\section{Acknowledgments}

Financial support by Dirección General de Enseñanza Superior, Spain (Project PB950371) is gratefully acknowledged.

\section{References}

[1] Debney G C, Kerr R P and Schild A 1969 J. Math. Phys. 101842

[2] Hauser I 1974 Phys. Rev. Lett. 33 1112; 33 1525(E) 
[3] Hauser I 1978 J. Math. Phys. 19661

[4] Ernst F J and Hauser I 1978 J. Math. Phys. 191816

[5] Halford W D 1979 J. Math. Phys. 201115

[6] Collinson C D 1980 J. Math. Phys. 212601

[7] McIntosh C B G 1985 Class. Quantum Grav. 287

[8] Stephani H and Herlt E 1985 Class. Quantum Grav. 2 L63

[9] Chinea F J 1988 Class. Quantum Grav. 5135

[10] Chinea F J 1988 Phys. Rev. D 373080

[11] Plebański J F and Przanowski M 1991 Phys. Lett. 152A 257

[12] Herlt E 1991 Gen. Rel. Grav. 23477

[13] Ludwig G and Yu Y B 1992 Gen. Rel. Grav. 2493

[14] Stephani H 1993 Class. Quantum Grav. 102187

[15] Finley J D III, Plebański J F and Przanowski M 1994 Class. Quantum Grav. 11157

[16] Hoenselaers C, Ferwagner R and Schief W 1994 Relativity Today, Proc. 4th Hungarian Relativity Workshop ed R P Kerr and Z Perjés (Budapest: Akadémiai Kiadó) p 47

[17] Ludwig G and Edgar S B 1996 Gen. Rel. Grav. 28707

[18] Edgar S B and Ludwig G 1997 Gen. Rel. Grav. 2919

[19] Finley J D III, Plebański J F and Przanowski M 1997 Class. Quantum Grav. 14489

[20] Chinea F J 1984 Phys. Rev. Lett. 52322

[21] Chinea F J and Fernández-Jambrina L 1997 in preparation 